\title{
Spatio-temporal evolution of ultrashort pulses in graded-index multimode fiber at normal dispersion
}

\author{
Ŭ̆ur Teğgin ${ }^{1,2}$ and Bülend Ortaç ${ }^{1,2}$ \\ 1. National Nanotechnology Research Center, Bilkent University, 06800 Bilkent, Ankara, Turkey \\ 2. Institute of Material Science and Nanotechnology (UNAM), Bilkent University, 06800, Bilkent, Ankara, Turkey
}

A novel nonlinear phenomenon at normal dispersion regime inside of graded-index multimode fiber (GRIN MMF), geometric parametric instability (GPI), can be observed while propagating beam experiences spatiotemporal evolution. Longhi theoretically predicted this GPI and associated with periodic refocusing inside GRIN MMF [1]. Later his work is verified by Krupa et al. [2] and Lopez-Galmiche et al. [3] and discrete GPI sidebands are reported with more than $120 \mathrm{THz}$ detuning from to pump frequency. These studies focused on quasicontinues pulse evolution in GRIN MMF and the spatio-temporal evolution of femtosecond pulses at normal dispersion regime remains unknown. Here, we present the first experimental observation of GPI sidebands with ultrashort pulses in GRIN MMF. To gain detailed understanding, we perform numerical simulations and theoretical calculations and obtained results are well-aligned with experimental observations.

In experiments, we use an amplified Ti:Sapphire laser as a pump source to generate linearly polarized, singlemode, 200 femtosecond ultrashort pulses at $800 \mathrm{~nm}$ with $1 \mathrm{kHz}$ repetition rate. A GRIN MMF with $50 \mathrm{~m}$ core diameter is excited with a lens and three-axis translation stage configuration (Fig.1.a). When we excite sufficient amount of high order modes with $354 \mathrm{~nJ}$ pump pulse, we obtain first GPI peak pair with $91 \mathrm{THz}$ frequency shift (Fig.1.b Inset). First Stokes and anti-Stokes peaks are observed around $1055 \mathrm{~nm}$ and $645 \mathrm{~nm}$ with $12 \mathrm{~nm}$ and 5 $\mathrm{nm}$ spectral bandwidths, respectively. To measure the beam quality of first Stokes peak, we separate it with a long pass filter and obtain a speckle-free, Gaussian-like near-field beam profile. Commonly used theoretical calculation [1,2,3] suggests that $\sim 93 \mathrm{THz}$ frequency detuning is expected for first GPI peak pair when pump pulses are selected at $800 \mathrm{~nm}$. To understand the spatio-temporal evolution of pump pulses, we also perform numerical simulations by coupled-mode analysis method [4]. Even though GRIN MMF supports hundreds of modes at $800 \mathrm{~nm}$, to reduce computation time, we perform a simulation with first three cylindrically symmetric modes and obtain similar results with $\sim 88 \mathrm{THz}$ frequency detuning for first GPI pair (Fig.1.b).

(a)
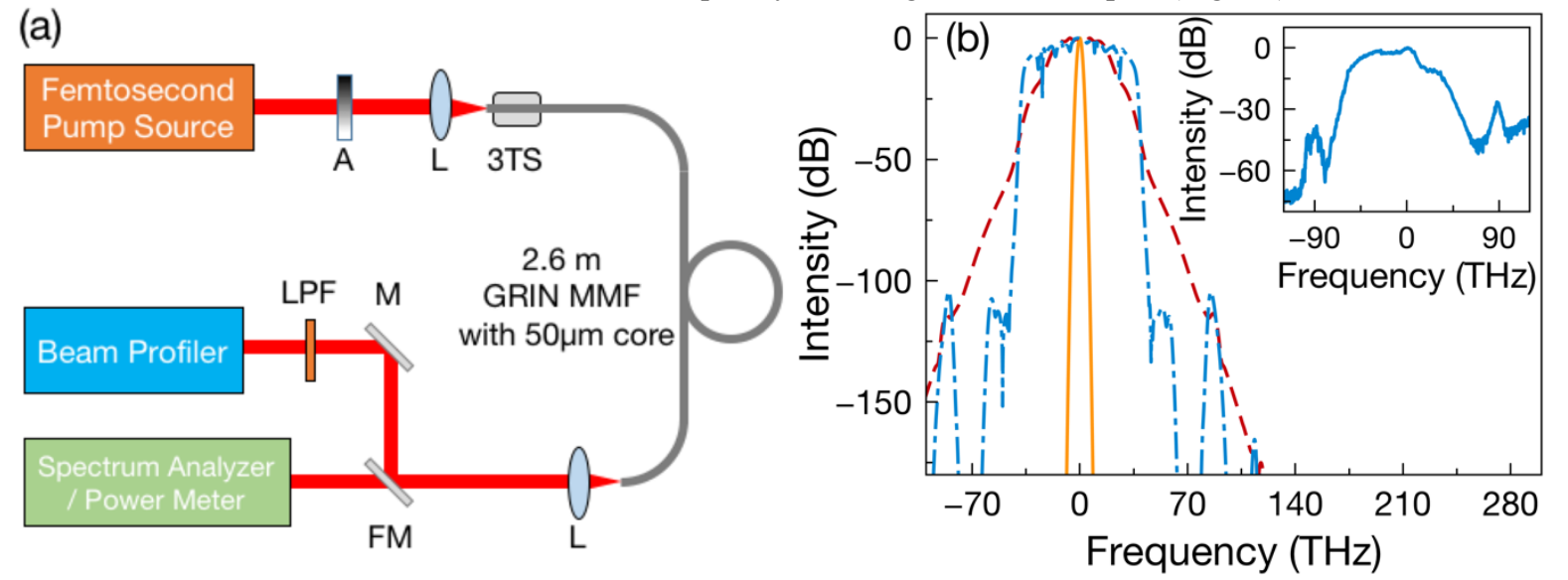

Fig. 1 (a) Schematic of the setup: A, attenuator; L, lens; 3TS, three-axis translation stage; FM, flipped mirror; M, mirror; LPF, long pass filter. (b) Simulation results obtained with $200 \mathrm{fs}, 345 \mathrm{~nJ}$ pump pulse at $800 \mathrm{~nm}$. Spectrum of launch pulse (solid line), spectrum of after $5 \mathrm{~cm}$ propagation (dash line), spectrum of after $2.6 \mathrm{~m}$ (dash-dot line). Inset: Experimentally measured spectrum at the end of $2.6 \mathrm{~m}$ GRIN MMF obtained with $345 \mathrm{~nJ}$ pump pulses.

In conclusion, we study the spatio-temporal evolution of femtosecond pulses in GRIN MMF experimentally and numerically. Our results present the first demonstration of GPI sidebands generation with ultrashort pulses in the literature. Simplified numerical and theoretical models are verified the experimental observations. With unique frequency shift, GPI can be used as new wavelength generation method. Obtained frequency shifts are depending on the wavelength of the pump pulses thus it can be tuned for desired applications.

\section{References}

[1] S. Longhi, "Modulational instability and space-time dynamics in nonlinear parabolic-index optical fibers," Opt. Lett. 28, 2363 (2003).

[2] K. Krupa, A. Tonello, A. Barthélémy, V. Couderc, B. M. Shalaby, A. Bendahmane, G. Millot and S. Wabnitz "Observation of geometric parametric instability induced by the periodic spatial self-imaging of multimode waves," Phys. Rev. Lett. 116, 183901 (2016).

[3] G. Lopez-Galmiche, Z. S. Eznaveh, M. A. Eftekhar, J. A. Lopez, L. G. Wright, F. Wise, D. Christodoulides, and R. A. Correa, "Visible supercontinuum generation in a graded index multimode fiber pumped at $1064 \mathrm{~nm}$," Opt. Lett. 41, 2553 (2016).

[4] A. Mafi, "Pulse propagation in a short nonlinear graded-index multimode optical fiber," Journ. Lightwave Technol. 30, 2803 (2012). 